\title{
Further evidence for a parent-of-origin effect at the NOP9 locus on language- related phenotypes
}

Kerry A. Pettigrew ${ }^{1 \dagger}$, Emily Frinton ${ }^{1 \dagger}$, Ron Nudel ${ }^{2}$, May T. M. Chan ${ }^{2,3}$, Paul Thompson ${ }^{4}$, Marianna E. Hayiou-Thomas ${ }^{5}$, Joel B. Talcott ${ }^{6}$, John Stein ${ }^{7}$, Anthony P. Monaco ${ }^{2}$, Charles Hulme ${ }^{8}$, Margaret J. Snowling ${ }^{4,9}$,

Dianne F. Newbury ${ }^{2}$ and Silvia Paracchini ${ }^{*}$

\begin{abstract}
Background: Specific language impairment (SLI) is a common neurodevelopmental disorder, observed in 5-10\% of children. Family and twin studies suggest a strong genetic component, but relatively few candidate genes have been reported to date. A recent genome-wide association study (GWAS) described the first statistically significant association specifically for a SLI cohort between a missense variant (rs4280164) in the NOP9 gene and language-related phenotypes under a parent-of-origin model. Replications of these findings are particularly challenging because the availability of parental DNA is required.

Methods: We used two independent family-based cohorts characterised with reading- and language-related traits: a longitudinal cohort ( $n=106$ informative families) including children with language and reading difficulties and a nuclear family cohort ( $n=264$ families) selected for dyslexia.

Results: We observed association with language-related measures when modelling for parent-of-origin effects at the NOP9 locus in both cohorts: minimum $P=0.001$ for phonological awareness with a paternal effect in the first cohort and minimum $P=0.0004$ for irregular word reading with a maternal effect in the second cohort. Allelic and parental trends were not consistent when compared to the original study.

Conclusions: A parent-of-origin effect at this locus was detected in both cohorts, albeit with different trends. These findings contribute in interpreting the original GWAS report and support further investigations of the NOP9 locus and its role in language-related traits. A systematic evaluation of parent-of-origin effects in genetic association studies has the potential to reveal novel mechanisms underlying complex traits.
\end{abstract}

Keywords: Language impairment, Dyslexia, Genetic association, Parent-of-origin, Candidate gene

\section{Background}

Specific language impairment (SLI) is a common neurodevelopmental disorder, estimated to affect $5-10 \%$ of preschool-aged children [1]. It is characterised by challenges with oral language acquisition, both in terms of the grammatical, syntactical and semantic aspects of speech production (expressive language) and in understanding the words of others (receptive language) [2]. An SLI diagnosis excludes other causes for language

\footnotetext{
* Correspondence: sp58@st-andrews.ac.uk

${ }^{\dagger}$ Equal contributors

${ }^{1}$ School of Medicine, University of St Andrews, St Andrews KY16 9TF, UK Full list of author information is available at the end of the article
}

impairment such as general cognitive and neurological problems and inadequate educational opportunities [3].

A strong genetic component has been suggested by family studies, with up to one third of affected children having parents with language or literacy impairment [4]. This observation is well supported by twin studies of school-aged children, suggesting heritability to be $50 \%$ or higher depending on diagnostic criteria $[5,6]$. Linkage analyses have identified at least five SLI loci $[7,8]$ : 2q36 (SLI5, OMIM \#615432), 7q36 (SLI4, OMIM \#612514), 13q21 (SLI3, OMIM \#607134), 16q24 (SLI1, OMIM \#606711) and 19q13 (SLI2, OMIM \#606712). Candidate genes within these regions include CMIP and $A T P 2 C 2$ at 
16q24 [9], CNTNAP2 at 7q36 [10] and TM4SF20 at 2q36.3 [11].

SLI presents significant comorbidity with other neurodevelopmental disorders, particularly with dyslexia [3]. Dyslexia (reading disability; RD) is a specific impairment in learning to read, affecting approximately $10 \%$ of children. SLI and RD present comorbidity in $43-55 \%$ of cases $[12,13]$, and it is likely that shared biology contributes to both disorders. The hypothesis is supported by twin [14] and family studies [15], which indicate that common environmental and genetic influences contribute to variation in language and reading abilities. Specific genetic factors have been found to be associated with both disorders. For example, the KIAA0319 dyslexia candidate has shown association with language skills in independent studies $[16,17]$. Candidate genes for dyslexia and SLI have been shown to affect language skills in individuals with autism spectrum disorder [18].

Genome-wide association studies (GWASs) for language phenotypes have been sparse so far and have been less successful when compared to results obtained for other disorders (http://genome.gov/gwastudies/). One of the main limitations is the availability of sufficiently large and well-characterised cohorts [8]. The only significant GWAS finding for language abilities has been reported for the $\mathrm{ROBO} 2$ gene through the analysis of epidemiological samples [19]. Other GWASs have used principal components of language ability within mixeddisorder cohorts but failed to detect statistically significant associations [20].

The first GWAS conducted specifically in an SLI cohort was reported recently for a cohort of 278 families [21]. Although child-based analyses did not reach significance, compelling findings were observed when modelling for parent-of-origin effects. A parent-oforigin effect is detected when an allelic influence on a trait depends on which parent a particular allele was inherited from. The most obvious explanatory mechanism would be imprinting through epigenetics. Imprinted genes are important for different aspects of brain development. Neurodevelopmental disorders, such as Prader-Willi syndrome and Angelman syndrome, which both present language deficits, involve the imprinted genomic regions on chromosome $15 \mathrm{q}$ (reviewed by Chamberlain and Lalande [22]). Recently, we described the first case of a deletion at the same chromosome 15 locus resulting in language impairment only, with no other syndromic manifestations [23]. It has been shown that non-imprinted genes in the mouse may still display parent-of-origin effects because of their interaction with imprinted loci and that this phenomenon is likely to be an overlooked factor contributing to complex traits [24]. The
GWAS that detected parent-of-origin effects for language impairment [21] used a categorical definition of SLI based on a low score (1.5 SD, or more, below the general population mean for their age) on expressive and/or receptive subtests of the Clinical Evaluation of Language Fundamentals (CELF) [25]. A variant in NOP9 on chromosome 14 was significantly associated (rs4280164, $P=3.74 \times 10^{-8}$ ) with paternal parent-oforigin effects. The strongest maternal effect was observed for an intergenic variant on chromosome 5 p13 but did not reach statistical significance within a GWAS context $\left(\mathrm{rs} 10447141, \quad P=1.16 \times 10^{-7}\right)$. The association on chromosome 5 was followed up in a sample of 313 language-impaired children and their mothers selected from the Avon Longitudinal Study of Parents and Children (ALSPAC) cohort. While association under a maternal parent-of-origin effect was observed at this locus, the opposite allelic trend was seen. The paternal effect for NOP9 was not followed up in the ALSPAC cohort since paternal DNA was unavailable. Replicating these associations is particularly challenging because testing parent-oforigin effects require parental DNA, which are not normally available in a case-control study design.

Here, we report the first follow-up study for the results of this GWAS for language impairment using a longitudinal cohort of 106 informative families well characterised with language-related phenotypes (332 total individuals, including parents and siblings). Given the overlap between SLI and dyslexia, we tested for association also with reading measures and analysed an additional cohort of 264 families selected primarily for dyslexia. Analysis of quantitative measures of reading and language ability, under a parent-of-origin model, showed statistically significant associations for rs4280164 at the NOP9 locus in both cohorts but with different allelic trends and parental effects.

\section{Methods \\ Study participants}

We investigated a longitudinal cohort (referred to as the York cohort), consisting of 106 informative families characterised with reading and language measures [26]. The probands included children with a family history of dyslexia $(n=46)$, children with preschool speech and/or language difficulties $(n=26)$ and typically developing children $(n=41)$. DNA from additional family members generated a total sample size of 332 individuals. Exclusion criteria were a non-white European ethnicity and/or a non-verbal IQ score below 70. Any child with a diagnosis of Attention Deficit Hyperactivity Disorder (ADHD) was excluded from the cohort prior to sample collection. Saliva samples were extracted from children and parents 
using Oragene kit (prepITL2P) (DNA Genotek, Ottawa, Canada).

The York cohort included a significant proportion of children with a family history of reading difficulties in addition to children with language problems. Accordingly, we analysed an additional cohort selected for a dyslexia diagnosis. This 'dyslexia cohort', composed of nuclear families, has been described previously $[27,28]$. Even if formal SLI diagnoses were not given to the participants of this cohort, it likely included children with language impairment given the significant comorbidity of this condition with dyslexia. The sample comprised 264 families for a total of 1037 individuals. Proband exclusion criteria included a non-white European origin, total IQ $<85$ and signs of other specific neurological conditions. DNA was obtained from a mix of blood and buccal swabs and extracted using standard procedures.

DNA was not available for 39 of the 212 (18 \%) and 59 of the $528(11 \%)$ parents in the York and dyslexia cohorts, respectively.

\section{Phenotype selection}

From the range of available language measures characterising the York cohort, we selected those most closely related to traits previously used in genetic analyses for language- and reading-related traits [17, 29-31]. These included the age at which first words are spoken (age of first words, AFW) and three core language measures from time-point 1 of the study: the ability to repeat nonwords (NWR [32]), and two expressive language measures assessed by the ability to name objects (CELFa [33]) and the ability to match spoken sentences to pictures (CELFb [33]). Three factor scores for speech (SPEECH), vocabulary (VOC) and grammar (GRAM) were also used to capture performance across language domains and were calculated for tests collected at preschool (ps) or school (sc) age. Because factor scores captured the longitudinal dimensions, all individual measures refer to the first collection in chronological time if the test was repeated at multiple times during the project. The correlation across language measures ranged between 0.37 and 0.45 for individual measures of vocabulary, grammar and phonology at time 1 (3-4 years old). Correlations between factor scores were higher; $r=0.8$ between vocabulary and grammar at school age.

At later ages ( $51 / 2$ and $61 / 2$ years old), reading measures included single-word reading (READ), Test of Word Reading Efficiency (TOWRE) [34], Wechsler Individual Achievement Test spelling (WIAT-SPELL) [35], letter writing (LW), letter sound knowledge (LSK), phonological awareness measured by a phoneme isolation task (PA-is), rapid automatic naming (RAN), non- word reading (PD) and a factor score for literacy (LIT). The correlation across reading and related measures ranged between 0.5 and 0.7 at age $5 \frac{1}{2}$ years; at $61 / 2$ years, the correlation between reading and both the vocabulary and the grammar factor scores was 0.5 (see Hulme et al. [36] for details of the longitudinal relationships between these measures).

In contrast to the York cohort, which included longitudinal measures, the dyslexia cohort was assessed only once for study participants with an age range of 6 to 25 years and language was not measured. We analysed the reading-related measures described previously in genetic studies of this cohort [37]. This included single-word reading (READ) and spelling (SPELL), tests for orthographic coding by an irregular word task (OC-irreg) and forced choice task (OCchoice; a pseudo-homophone detection task), phoneme awareness (PA) measured by the spoonerism test, and PD. These measures show correlation with one another in the range of $0.41-0.76$ [37]. The only measure directly comparable between the York and the dyslexia cohorts is READ, whereas PA, although assessing the same trait, was measured using different tests.

\section{Genotyping and statistical analysis}

Markers rs4280164 (NOP9, chromosome 14) and rs10447141 (on 5p13) were genotyped using TaqMan assays (LifeTechnologies, Paisley, UK) on a ViiA7 qPCR instrument (LifeTechnologies, Paisley, UK) in the York cohort, and as part of a multiplex Sequenom assay in the dyslexia cohort. All assays passed standard quality control tests for call rate, Mendelian error and HardyWeinberg equilibrium. Furthermore, genome-wide genotyping data, which were available for a subset of the siblings (York, $n=94$; dyslexia, $n=758$ ), showed a $100 \%$ concordance with genotypes generated for the entire cohorts in this study. Principal component analysis (PCA) of the genome-wide genotyping data was used to assess population stratification. All samples retained for the analysis showed no evidence of population stratification, thus allowing for a total association model, within and across families.

Statistical analysis of quantitative family data was conducted using MERLIN [38] and QTDT [39]. MERLIN was used to further control for genotyping errors and to estimate identity-by-descent (IBD) sharing scores. Parent-of-origin analysis was conducted in QTDT under the total association model (-wega -at) testing separately for maternally (-om) or paternally (-op) derived alleles and to determine whether maternal and paternal alleles were significantly different (-ot). The informative individuals used by QTDT under this model derive from two groups. The first group includes those individuals 
where both parents are genotyped and where one parent is homozygous or the mother and father have different genotypes. When paternal or maternal imprinting is tested, the father or the mother, respectively, must be heterozygous. The second group includes all individuals with at least one homozygous parent. The number of informative individuals in our analysis was 107 (rs10447141) and 111 (rs4280164) in the York cohort. In the dyslexia cohort, the informative individuals ranged from 443 to 470 (rs10447141) and 462 to 491 (rs4280164) depending on the amount of missing genotype or phenotypic data.

In total, we tested two markers for 25 phenotypes resulting in 50 different tests. Therefore, by applying a Bonferroni correction for multiple testing, statistical significance is reached by $P$ values $<0.001$. This is a conservative and stringent correction as the phenotypes were correlated with one another and are not independent.

\section{Results}

We conducted a replication study for the NOP9 and 5 p13 variants implicated in SLI susceptibility through a recent GWAS, which incorporated parent-of-origin information in the analytical model [21]. We performed quantitative association analyses with a range of language/reading-related phenotypes in two distinct cohorts. We investigated a longitudinal cohort of 315 individuals from 106 families enriched for language impairment and family history of dyslexia (York cohort). The rs4280164 and rs10447141 markers, representing the top associated variants at the NOP9 and $5 \mathrm{p} 13$ loci [40], respectively, were tested for association with quantitative phenotypes. Allele frequencies (Table 1) were comparable with HapMap data for populations of European origin (HapMap minor allele frequency (MAF): rs4280164 $=18 \%$ and $r s 10447141=29 \%$ ), although rs4280164 MAF was higher in our cohorts (22-24\%). The strongest association signal was observed between rs4280164 and age at first word (AFW) $(P=0.003$; Table 1). The only other association was observed for rs10447141 and PA $(P=0.01)$.

When parent-of-origin was incorporated into the analysis, we observed association for a range of measures but with an inconsistent pattern. The original study

Table 1 Association analysis result summary

\begin{tabular}{|c|c|c|c|c|c|c|c|c|c|c|}
\hline \multirow[t]{2}{*}{ Cohort } & \multicolumn{2}{|l|}{ Phenotype } & \multirow[b]{2}{*}{ SNP } & \multirow[t]{2}{*}{ MAF } & \multicolumn{2}{|c|}{ Standard $^{a}$} & \multicolumn{4}{|c|}{ Parent of origin } \\
\hline & Trait & Test & & & $\begin{array}{l}\text { Risk } \\
\text { allele }\end{array}$ & $\begin{array}{l}P \\
\text { value }\end{array}$ & $\begin{array}{l}\text { Risk } \\
\text { allele }\end{array}$ & $\begin{array}{l}P \text { value } \\
\text {-om/-op }\end{array}$ & $\begin{array}{l}\text { Parental } \\
\text { effect }\end{array}$ & $\begin{array}{l}P \\
\text { value } \\
\text {-ot }\end{array}$ \\
\hline \multirow[t]{12}{*}{ York $N=106$ families } & Language & AFW & rs4280164 & A (24\%) & G & 0.003 & G & 0.02 & M & \\
\hline & & NWR & & & & & G & 0.002 & & 0.004 \\
\hline & & CELFa & & & & & G & 0.03 & & \\
\hline & & CELFb & & & & & G & 0.02 & & 0.009 \\
\hline & & SPEECH (ps) & & & & & G & 0.03 & & \\
\hline & & SPEECH (sc) & & & & & G & 0.02 & & 0.04 \\
\hline & & GRAM (ps) & & & & & G & 0.04 & & \\
\hline & & LW & & & & & G & 0.04 & & \\
\hline & Reading & PA-is & & & & & A & 0.001 & $P$ & 0.003 \\
\hline & & RAN (ps) & & & & & A & 0.04 & & 0.01 \\
\hline & Reading & PA-is & rs10447141 & T (29\%) & C & 0.01 & & & & \\
\hline & & LSK & & & & & C & 0.04 & $P$ & 0.02 \\
\hline \multirow[t]{4}{*}{ Dyslexia $N=264$ families } & Reading & READ & rs4280164 & A (22\%) & & & A & 0.008 & M & 0.03 \\
\hline & & OC-irreg & & & & & A & 0.0004 & & 0.0053 \\
\hline & & PD & & & $A$ & 0.045 & A & 0.003 & & 0.02 \\
\hline & & PA & & & & & A & 0.01 & & 0.03 \\
\hline \multirow[t]{2}{*}{ Nudel et al. $(2014)^{\mathrm{b}} N=278$ probands } & Language & SLI & rs4280164 & & & & G & $3.74 \times 10^{-8}$ & $P$ & \\
\hline & & & rs10447141 & & & & C & $1.16 \times 10^{-7}$ & $M$ & \\
\hline
\end{tabular}

Abbreviations: MAF minor allele frequency calculated among parents, AFW age at first word, NWR non-word repetition, CELFa Clinical Evaluation of Language Fundamentals (expressive vocabulary), CELFb Clinical Evaluation of Language Fundamentals (sentence structure), SPEECH speech factor score, GRAM language grammar factor score, $L W$ letter writing, $P A$ phonological awareness-phoneme isolation, $R A N$ rapid automatic naming, $L S K$ letter sound knowledge, $R E A D$ singleword reading, $O C$-irreg orthographic coding by an irregular word task, $p s$ preschool, sc school, $M$ maternal, $P$ paternal Only tests leading to $P$ values $<0.05$ are reported and $P$ values $<0.001$ ( $=a$ adjusted for multiple testing) are in italics ${ }^{a}$ Association analysis not modelled for parent of origin

${ }^{\mathrm{b}}$ Analysis was conducted for a categorical definition of SLI and not for quantitative measures 
reported a paternal effect at rs4280164 where the G allele was associated with an SLI status [21]. In contrast, we observed a maternal effect for the $G$ allele across a range of language measures and a paternal effect for the A allele for some reading measures (Table 1).

In the parent-of-origin model, the association with AFW was attenuated $(P=0.02)$ but strongest associations were observed for NWR $(P=0.002$; maternal; risk for $\mathrm{G}$ allele) and PA-is ( $P=0.001$; paternal; risk for A allele). The rs10447141 marker showed only marginally significant associations for LSK under a paternal origin effect $(P=0.04)$.

Given the associations we observed for PA-is in the York cohort, which included a significant proportion of children with a family history of reading difficulties, we also investigated 264 families from the 'dyslexia cohort'. Association analysis under a standard model showed only a marginally significant association for rs4280164 with PD $(P=0.045)$.

When association analysis was conducted under a parent-of-origin model, rs4280164 showed association for most of the available reading measures with the strongest signal observed for OC-irreg $(P=0.0004)$. The trend of association was also inconsistent with previous observation; A was the risk allele under a maternal effect. No association was observed for rs10447141 with standard or parent-of-origin models in the dyslexia cohort. Paternal and maternal effects were confirmed to be statistically independent (see ' $P$ value -ot' column in Table 1: association was detected when testing both maternal and paternal alleles in the same test) for most measures that showed parent-of-origin effects.

Although the phenotypes are correlated with each other and cannot be considered independent, our results need to be interpreted in the light of multiple testing. Two associations reached the significance level adjusted for multiple test comparisons ( $\alpha=0.001$ for $N=50$ tests) under a conservative assumption of independence across all tests: PAis in the York cohort ( $P=0.001$; paternal; risk for A allele) and OC-irreg in the dyslexia cohort $(P=0.0004$; maternal; risk for A allele).

\section{Discussion}

We report the first follow-up analysis of a GWAS implicating two loci, one on chromosome 14 (rs4280164, NOP9 gene) and another on chromosome 5 (rs10447141), in language impairment susceptibility under a parent-oforigin model [21]. We utilised two cohorts characterised with quantitative measures and for which parental DNA was available. The York cohort is a longitudinal study designed to investigate language and literacy development, while the dyslexia cohort was established in order to investigate the genetic component of dyslexia. The significant comorbidity between SLI and dyslexia prompted us to include reading measures in the analysis. Marker rs10447141, which did not reach statistical significance in the original study [21], only showed marginal association in the York cohort. Marker rs4280164 in the NOP9 gene yielded significant associations when modelling for parent-of-origin across a range of measures in both cohorts but with inconsistent allelic and parental effects. The original study reported that risk was conferred by the major allele (G) with a paternal effect. Conversely, the A allele showed association in the dyslexia cohort but with a maternal effect. The results in the York cohort are intriguing: the $\mathrm{G}$ allele was associated with poor language skills with a maternal effect, whereas a paternal effect for the A allele was observed with some reading measures. While it would be tempting to speculate that different alleles affect different traits (e.g. reading or language) under different parental effect, caution should be used in interpreting the results until further replication analyses are conducted. It is also worth noting that opposite allelic trends were observed for the association described at the chromosome 14 loci in the two cohorts (i.e. a maternal effect was observed in both the SLI cohort and ALSPAC languageimpaired subgroup but with opposite allelic effects) tested in the original study [21]. Recently, re-analysis of the SLI cohort with an improved version of the EMIM software which could support parent-of-origin analyses in a larger number of families showed a strong association for rs4280164 and decreased signal for rs10447141 in line with our findings [41]. The strong associations in the discovery study could have been an over-estimation because of the well-established phenomenon known as 'winner's curse' [42], and we thus expected smaller effect sizes in this replication study.

The differences in parent-of-origin could be intrinsic to our study design and be the result of random fluctuation due to small sample sizes. The original study included 278 affected children, and the present study was based on cohorts of 106 and 264 families leading to a range of 107 to 111 and 443 to 491 informative individuals, respectively. Undoubtedly, sample size is a limitation of the present study, especially in the York cohort, which could explain the inconsistent trend. However, the strongest association was observed in the largest cohort (e.g. dyslexia cohort) for the OC-irreg phenotype $(P=0.0004$; maternal; risk for A allele). The rs4280164 minor allele (A) has a frequency of $22-24 \%$ (18 \% reported in HapMap for European population) and corresponds to a missense variant leading to a substitution $(\mathrm{S} 308 \mathrm{~N})$ in the protein encoded by NOP9. The minor allele, which gave the strongest associations in both the York and dyslexia cohorts (Table 1), was also predicted to have a damaging effect on protein function [21]. This substitution falls in a highly conserved sequence of the protein in proximity to RNA-binding domains that 
characterise the main function of NOP9 [21]. While a change in the protein sequence is the most obvious effect of this SNP, the rs4280164 itself is also listed as one of the most significant markers having an expression quantitative trait locus (eQTL) effect in the GTEx database (http://www.gtexportal.org/home/). An eQTL effect is reported across different tissues, the strongest of which $(P=3.8 \mathrm{e}-20$, effect size $=-0.55)$ is observed for the neighbouring $L T B 4 R$ gene in the thyroid. This observation does not offer an immediate interpretation of how the SNP could affect language-related phenotype; however, it is worth noting that eQTL databases depend on the available tissues and the foetal brain, which would be the most relevant to our phenotype of interest, is not immediately accessible.

Differences in study design also have the potential to affect the results. While we tested for association with various quantitative phenotypes, the original study applied a categorical definition of SLI [21]. Further differences between the studies are represented by the use of different tests in the York and dyslexia cohorts, even when measuring the same trait. For example, phonological awareness, which showed association in both cohorts, was assessed by the phoneme isolation task in the York cohort and with the spoonerism test in the dyslexia cohort. Some of the differences in the tests used are also dependent on the age of the study participants. Participants in the York cohort were assessed mainly during preschool years, while participants in the dyslexia cohort were recruited after they started attending school and experiencing reading problems. The dyslexia cohort was not characterised with oral language measures. Lack of homogeneity for the phenotypes used in genetic studies is increasingly recognised as a major challenge in advancing the field [8].

Our findings could also reflect the complex nature of the mechanisms underlying parent-of-origin effects that can result from different phenomena. Imprinting has been demonstrated for less than $1 \%$ human genes, but its contribution to trait variation could be higher than expected [43]. While there has not been direct evidence that NOP9 is imprinted, it has been suggested that its location overlaps with potentially imprinted regions on chromosome 14 [41, 44, 45]. Studies that implemented parent-of-origin in their models are increasingly identifying a significant contribution of parental effects to different traits such as cancer [46], diabetes [46, 47], body mass index (BMI) [48] and pubertal timing [49]. Recently, it has been shown that an unexpectedly large number of autosomal genes $(N=4227)$ present a monoallelic expression and a large proportion of those contribute to the variability of human traits, but NOP9 was not tested in this study [50]. Parent-of-origin effect could be the result of an interaction between genes in imprinted region and other loci, as shown recently in mouse models [24], leading to heterogeneous and complex association patterns. Parent-of-origin evaluation might therefore explain part of the so-called missing heritability [24]. It has been consistently reported that genetic associations reported so far are only able to account for a small proportion of the estimated heritability [51]. The remaining unexplained heritability, or missing heritability, is a phenomenon observed for all complex traits and disorders, even in well-powered samples. Rare variants identified through exome and whole genome sequencing studies certainly contribute to explaining part of the missing heritability, but it is clear that other factors need to be taken into account.

Therefore, while we cannot exclude that the association patterns we report are due to chance effects, our results encourage further investigations of the NOP9 locus in the context of language abilities.

\section{Conclusions}

In summary, we report a follow-up study for the first GWAS for SLI conducted under a parent-of-origin model by testing the top two associated markers [21]. Our study detected a parent-of-origin effect with one of these markers at the NOP9 locus for both language and reading-related phenotypes in two independent cohorts. Although allelic and parental trends are not in line with the findings of the original GWAS, the results suggest that this locus might be implicated in neurodevelopmental phenotypes through a complex parental inheritance mechanism. To elucidate such mechanisms, it will be necessary to further study parent-of-origin effects at this locus in additional cohorts selected for reading and language impairment. Our study, once again, highlights the difficulties in conducting replication studies across heterogeneous datasets; therefore, it will be important to define common core tests that would permit direct comparison of different cohorts. We propose that parent-oforigin effects should be evaluated more systematically in association studies for language disorders as well as other complex traits since this model has the potential to elucidate some of the missing heritability.

\footnotetext{
Acknowledgements

SP is a Royal Society University Research Fellow. Support to the analysis was provided by the St Andrews Bioinformatics Unit funded by the Wellcome Trust [grant 097831/Z/11/Z]. Analysis of the cohort was supported by a Wellcome Trust Programme Grant to MJS [WT082032MA]. EF is the recipient of a Wolfson Intercalated Award. MTMC is the recipient of the Wilkinson Junior Research Fellowship at Worcester College, Oxford, and was funded by the Esther Yewpick Lee Millennium Scholarship. RN was funded by a University of Oxford Nuffield Department of Medicine Prize Studentship. DFN is an MRC Career Development Fellow. The work of the Newbury lab is funded by the Medical Research Council [G1000569/1 and MR/J003719/1]. The work of the Wellcome Trust Centre in Oxford is supported by the Wellcome Trust [090532/Z/09/Z].
} 


\section{Competing interests}

The authors declare that they have no competing interests.

\section{Authors' contributions}

$K P, E F, R N$ and MTM generated and/or analysed the data; PT, EH-T, JBT, JS, $\mathrm{APM}, \mathrm{CH}$ and MJS contributed in acquiring the data; KP, EF, RN, EH-T, MJS and DFN contributed in interpreting the data; SP and DFN conceived and designed the study; KP, EF and SP wrote the first draft of the manuscript; all authors revised the manuscript and contributed critically to it. All authors read and approved the final manuscript.

\section{Ethics approval and consent to participate}

The study was approved by Yorkshire \& The Humber Bridge-Humber Bridge NHS Research Ethics, the Ethics Committee at the University of York Department of Psychology and the Oxfordshire Psychiatric Research Ethics Committee (OPREC O01.02). Informed consent was obtained from all participants, or from their carers, included in the study.

\section{Author details \\ ${ }^{1}$ School of Medicine, University of St Andrews, St Andrews KY16 9TF, UK. ${ }^{2}$ Wellcome Trust Centre for Human Genetics, University of Oxford, Oxford OX3 7BN, UK. ${ }^{3}$ Worcester College, University of Oxford, Oxford OX1 2HB, UK ${ }^{4}$ Department of Experimental Psychology, University of Oxford, South Parks Road, Oxford OX1 3PT, UK. ${ }^{5}$ Department of Psychology, University of York, York YO10 5DD, UK. ${ }^{6}$ School of Life and Health Sciences, Aston University, Birmingham B4 7ET, UK. ${ }^{7}$ Department of Physiology, University of Oxford, Parks Road, Oxford OX1 3PT, UK. ${ }^{8}$ Division of Psychology and Language Sciences, University College London, London WC1 3PG, UK. 'St John's College, University of Oxford, Oxford OX1 3JP, UK.}

\section{Received: 7 December 2015 Accepted: 3 June 2016}

Published online: 14 June 2016

\section{References}

1. Tomblin JB, Records NL, Buckwalter P, Zhang X, Smith E, O'Brien M. Prevalence of specific language impairment in kindergarten children. J Speech Lang Hear Res. 1997;40(6):1245-60.

2. Pennington BF, Bishop DV. Relations among speech, language, and reading disorders. Annu Rev Psychol. 2009;60:283-306. doi:10.1146/annurev.psych.60. 110707.163548.

3. Bishop DV, Snowling MJ. Developmental dyslexia and specific language impairment: same or different? Psychol Bull. 2004;130(6):858-86.

4. Barry JG, Yasin I, Bishop DV. Heritable risk factors associated with language impairments. Genes Brain Behav. 2007;6(1):66-76. doi:10.1111/j.1601-183X. 2006.00232.x.

5. Bishop DV. The role of genes in the etiology of specific language impairment. J Commun Disord. 2002:35(4):311-28.

6. Bishop DV, Hayiou-Thomas ME. Heritability of specific language impairment depends on diagnostic criteria. Genes Brain Behav. 2008;7(3):365-72. doi:10. 1111/j.1601-183X.2007.00360.x.

7. Newbury DF, Monaco AP. Genetic advances in the study of speech and language disorders. Neuron. 2010;68(2):309-20. doi:10.1016/j.neuron. 2010.10.001

8. Newbury DF, Monaco AP, Paracchini S. Reading and language disorders: the importance of both quantity and quality. Genes (Basel). 2014;5(2):285-309. doi:10.3390/genes5020285.

9. Newbury DF, Winchester L, Addis L, Paracchini S, Buckingham LL, Clark A, et al. CMIP and ATP2C2 modulate phonological short-term memory in language impairment. Am J Hum Genet. 2009;85(2):264-72. doi:10.1016/j. ajhg.2009.07.004..

10. Vernes SC, Newbury DF, Abrahams BS, Winchester L, Nicod J, Groszer M, et al. A functional genetic link between distinct developmental language disorders. N Engl J Med. 2008;359(22):2337-45. doi:10.1056/NEJMoa0802828.

11. Wiszniewski W, Hunter JV, Hanchard NA, Willer JR, Shaw C, Tian Q, et al. TM4SF20 ancestral deletion and susceptibility to a pediatric disorder of early language delay and cerebral white matter hyperintensities. Am J Hum Genet. 2013;93(2):197-210. doi:10.1016/j.ajhg.2013.05.027.

12. McArthur GM, Hogben JH, Edwards VT, Heath SM, Mengler ED. On the "specifics" of specific reading disability and specific language impairment. J Child Psychol Psychiatry. 2000;41(7):869-74.
13. Snowling M, Bishop DV, Stothard SE. Is preschool language impairment a risk factor for dyslexia in adolescence? J Child Psychol Psychiatry. 2000;41(5):587-600.

14. Harlaar N, Hayiou-Thomas ME, Dale PS, Plomin R. Why do preschool language abilities correlate with later reading? A twin study. J Speech Lang Hear Res. 2008:51(3):688-705. doi:10.1044/1092-4388(2008/049).

15. Logan J, Petrill SA, Flax J, Justice LM, Hou L, Bassett AS, et al. Genetic covariation underlying reading, language and related measures in a sample selected for specific language impairment. Behav Genet. 2011;41(5):651-9. doi:10.1007/s10519-010-9435-0.

16. Rice ML, Smith SD, Gayan J. Convergent genetic linkage and associations to language, speech and reading measures in families of probands with specific language impairment. J Neurodev Disord. 2009;1(4):264-82. doi:10. 1007/s11689-009-9031-x.

17. Newbury D, Paracchini S, Scerri T, Winchester L, Addis L, Richardson AJ, et al. Investigation of dyslexia and SLI risk variants in reading-and languageimpaired subjects. Behav Genet. 2011;41(1):90-104.

18. Eicher JD, Gruen JR. Language impairment and dyslexia genes influence language skills in children with autism spectrum disorders. Autism Res. 2015;8(2):229-34. doi:10.1002/aur.1436.

19. St Pourcain B, Cents RA, Whitehouse AJ, Haworth CM, Davis OS, O'Reilly PF, et al. Common variation near $\mathrm{ROBO} 2$ is associated with expressive vocabulary in infancy. Nat Commun. 2014;5:4831. doi:10.1038/ncomms5831.

20. Gialluisi A, Newbury DF, Wilcutt EG, Olson RK, DeFries JC, Brandler WM, et al. Genome-wide screening for DNA variants associated with reading and language traits. Genes Brain Behav. 2014;13(7):686-701. doi:10.1111/gbb.12158.

21. Nudel R, Simpson NH, Baird G, O'Hare A, Conti-Ramsden G, Bolton PF, et al. Genome-wide association analyses of child genotype effects and parent-oforigin effects in specific language impairment. Genes Brain Behav. 2014; 13(4):418-29. doi:10.1111/gbb.12127.

22. Chamberlain SJ, Lalande M. Neurodevelopmental disorders involving genomic imprinting at human chromosome 15q11-q13. Neurobiol Dis. 2010;39(1):13-20. doi:10.1016/j.nbd.2010.03.011.

23. Pettigrew KA, Reeves E, Leavett R, Hayiou-Thomas ME, Sharma A, Simpson $\mathrm{NH}$, et al. Copy number variation screen identifies a rare de novo deletion at chromosome 15q13.1-13.3 in a child with language impairment. PLoS One. 2015;10(8):e0134997. doi:10.1371/journal.pone.0134997.

24. Mott R, Yuan W, Kaisaki P, Gan X, Cleak J, Edwards A, et al. The architecture of parent-of-origin effects in mice. Cell. 2014;156(1-2):332-42. doi:10.1016/j. cell.2013.11.043.

25. Semel EM, Wiig EH, Secord W. Clinical evaluation of language fundamentals—revised. San Antonio: Psychological Corporation; 1992.

26. Nash HM, Hulme C, Gooch D, Snowling MJ. Preschool language profiles of children at family risk of dyslexia: continuities with specific language impairment. J Child Psychol Psychiatry. 2013;54(9):958-68. doi:10.1111/jcpp.12091.

27. Scerri TS, Paracchini S, Morris A, MacPhie IL, Talcott J, Stein J, et al. Identification of candidate genes for dyslexia susceptibility on chromosome 18. PLoS One. 2010;5(10):e13712.

28. Francks C, Paracchini S, Smith SD, Richardson AJ, Scerri TS, Cardon LR, et al. A 77-kilobase region of chromosome 6p22.2 is associated with dyslexia in families from the United Kingdom and from the United States. Am J Hum Genet. 2004;75(6):1046-58. doi:10.1086/426404.

29. Alarcon M, Abrahams BS, Stone JL, Duvall JA, Perederiy JV, Bomar JM, et al. Linkage, association, and gene-expression analyses identify CNTNAP2 as an autism-susceptibility gene. Am J Hum Genet. 2008;82(1):150-9.

30. Whitehouse AJ, Bishop DV, Ang QW, Pennell CE, Fisher SE. CNTNAP2 variants affect early language development in the general population. Genes Brain Behav. 2011. doi:10.1111/j.1601-183X.2011.00684.x.

31. Bishop DV, North T, Donlan C. Nonword repetition as a behavioural marker for inherited language impairment: evidence from a twin study. J Child Psychol Psychiatry. 1996;37(4):391-403.

32. Seeff-Gabriel H, Chiat S, Roy P. The early repetition battery. London: Pearson Assessment; 2008

33. Wiig EH, Secord W, Semel E. Clinical evaluation of language fundamentals-Preschool. Second UK edition. London: Harcourt Assessment; 2006

34. Torgesen JK, Wagner RK, Rashotte CA. Test of word reading efficiency (TOWRE). Austin, TX: Pro-Ed; 1999.

35. Wechsler D. Wechsler individual achievement test. 2nd ed. San Antonio: The Psychological Corporation; 2002. 
36. Hulme C, Nash HM, Gooch D, Leavett R, Snowling MJ. Predictors of individual differences in the foundation of early literacy young children. Psychol Sci. 2015. (In press)

37. Marlow AJ, Fisher SE, Richardson AJ, Francks C, Talcott JB, Monaco AP, et al. Investigation of quantitative measures related to reading disability in a large sample of sib-pairs from the UK. Behav Genet. 2001;31(2):219-30.

38. Abecasis GR, Cherny SS, Cookson WO, Cardon LR. Merlin—rapid analysis of dense genetic maps using sparse gene flow trees. Nat Genet. 2002;30(1):97-101.

39. Abecasis GR, Cardon LR, Cookson WO. A general test of association for quantitative traits in nuclear families. Am J Hum Genet. 2000;66(1):279-92.

40. Nudel R, Simpson NH, Baird G, O'Hare A, Conti-Ramsden G, Bolton PF, et al. Associations of HLA alleles with specific language impairment. J Neurodev Disord. 2014;6(1):1. doi:10.1186/1866-1955-6-1.

41. Howey R, Mamasoula C, Topf A, Nudel R, Goodship JA, Keavney BD, et al. Increased power for detection of parent-of-origin effects via the use of haplotype estimation. Am J Hum Genet. 2015;97(3):419-34. doi:10.1016/j. ajhg.2015.07.016.

42. Zollner S, Pritchard JK. Overcoming the winner's curse: estimating penetrance parameters from case-control data. Am J Hum Genet. 2007;80: 605-15. doi:10.1086/512821.

43. Lawson HA, Cheverud JM, Wolf JB. Genomic imprinting and parent-oforigin effects on complex traits. Nat Rev Genet. 2013;14(9):609-17. doi:10.1038/nrg3543.

44. Kotzot D. Comparative analysis of isodisomic and heterodisomic segments in cases with maternal uniparental disomy 14 suggests more than one imprinted region. Clin Genet. 2001;60(3):226-31.

45. Sutton VR, Shaffer LG. Search for imprinted regions on chromosome 14: comparison of maternal and paternal UPD cases with cases of chromosome 14 deletion. Am J Med Genet. 2000;93(5):381-7.

46. Kong A, Steinthorsdottir V, Masson G, Thorleifsson G, Sulem P, Besenbacher $S$, et al. Parental origin of sequence variants associated with complex diseases. Nature. 2009;462(7275):868-74. doi:10.1038/nature08625.

47. Small KS, Hedman AK, Grundberg E, Nica AC, Thorleifsson G, Kong A, et al. Identification of an imprinted master trans regulator at the KLF14 locus related to multiple metabolic phenotypes. Nat Genet. 2011;43(6):561-4. doi: 10.1038/ng.833.

48. Hoggart CJ, Venturini G, Mangino M, Gomez F, Ascari G, Zhao JH, et al. Novel approach identifies SNPs in SLC2A10 and KCNK9 with evidence for parent-of-origin effect on body mass index. PLoS Genet. 2014;10(7): e1004508. doi:10.1371/journal.pgen.1004508.

49. Perry JR, Day F, Elks CE, Sulem P, Thompson DJ, Ferreira T, et al. Parent-oforigin-specific allelic associations among 106 genomic loci for age at menarche. Nature. 2014;514(7520):92-7. doi:10.1038/nature13545.

50. Savova V, Chun S, Sohail M, McCole RB, Witwicki R, Gai L, et al. Genes with monoallelic expression contribute disproportionately to genetic diversity in humans. Nat Genet. 2016;48(3):231-7. doi:10.1038/ng.3493.

51. Manolio TA, Collins FS, Cox NJ, Goldstein DB, Hindorff LA, Hunter DJ, et al. Finding the missing heritability of complex diseases. Nature. 2009:461(7265): 747-53. doi:10.1038/nature08494.

\section{Submit your next manuscript to BioMed Central and we will help you at every step:}

- We accept pre-submission inquiries

- Our selector tool helps you to find the most relevant journal

- We provide round the clock customer support

- Convenient online submission

- Thorough peer review

- Inclusion in PubMed and all major indexing services

- Maximum visibility for your research

Submit your manuscript at www.biomedcentral.com/submit

C Biomed Central 